\title{
Propiedades psicométricas del Inventario de Rahim en una muestra de adolescentes estudiantes de bachillerato
}

\section{Psychometric properties of the Rahim's Inventory in a sample of teenager high school students}

\author{
Alejandro César Antonio Luna-Bernal ${ }^{*}{ }^{1}$, Ana Cecilia Valencia-Aguirre ${ }^{1}$, José María Nava-Preciado ${ }^{1}$ \\ 1 - Universidad de Guadalajara, Departamento de Filosofia, México. \\ Introducción \\ Método \\ Resultados \\ Discusión \\ Recibido: 27/02/2018 Revisado: 18/05/2018 Aceptado: 28/05/2018 \\ Referencias
}

\section{Resumen}

El presente estudio analiza las propiedades psicométricas del Inventario de Rahim sobre Estilos de Manejo de Conflictos (ROCI-II, forma C; Rahim, 1983) en una versión adaptada al contexto de los conflictos entre adolescentes compañeros de aula en el bachillerato. La muestra estuvo compuesta por 663 estudiantes mexicanos con rango de edad de 15 a 19 años. Además del ROCI-II los participantes respondieron el Cuestionario sobre Estilos de Mensajes en el Manejo de Conflictos (CMMS) de Ross y DeWine (1988). Un análisis factorial confirmatorio (AFC) dio como resultado un moderado ajuste para un modelo tetrafactorial. Este estudio analiza posibles diferencias por sexo, edad y grado escolar. Se discuten estos y otros resultados en el marco de la literatura sobre estilos de manejo de conflictos en adolescentes.

Palabras clave: Conflictos escolares, estilos de manejo de conflictos, resolución de conflictos, modelo de doble preocupación, adolescentes

\begin{abstract}
This study analyzes the psychometric properties of an adapted version of the Rahim's Inventory on Conflict Management Styles (ROCI-II, Form C; Rahim, 1983), which evaluates conflict-handling styles in the context of conflicts among adolescent high school classmates. The sample was composed of 663 Mexican high school students from 15 to 19 years old. Participants answered the ROCI-II as well as the Ross-DeWine Conflict Management Message Style Instrument (CMMS; Ross \& DeWine, 1988). A confirmatory factor analysis (CFA) was performed, finding a moderate fit for a four-factor model. Possible differences by sex, age and school grade were analyzed. All these findings are discussed within the framework of the literature on conflict management styles in adolescents.
\end{abstract}

Key words: School conflict, conflict management styles, conflict resolution, dual concern model, adolescents 


\section{Introducción}

Los estilos de manejo de conflictos pueden definirse como las diversas orientaciones actitudinales y comportamentales que los individuos asumen frente a los conflictos interpersonales que se les presentan. Abas (2010) los entiende como "la manera en que nos aproximamos a la otra parte en una situación de conflicto" (p. 13). En la literatura académica, se han formulado diversos modelos para conceptualizar y evaluar estos estilos (Blake \& Mouton, 1970; Kimsey \& Fuller, 2003; Rahim, 1983; Ross \& DeWine, 1988; Thomas \& Kilmann, 1974).

En España y Latinoamérica, los instrumentos más empleados para evaluar estilos de manejo de conflictos en muestras de adolescentes en el contexto escolar han sido las versiones traducidas y adaptadas del Conflict Management Message Style Instrument (CMMS) de Ross y DeWine (1988; Luna \& Laca, 2014; Luna-Bernal \& De Gante-Casas, 2017; Luna-Bernal, Mejía-Ceballos \& Laca-Arocena, 2017) y del cuestionario Conflictalk de Kimsey y Fuller (2003; Garaigordobil \& Maganto, 2011; Garaigordobil, Machimbarrena, \& Maganto, 2016; Laca, Alzate, Sánchez, Verdugo, \& Guzmán, 2006).

Cabe mencionar que tanto el CMMS como el Conflictalk son instrumentos que están basados en un modelo que contempla tres estilos de manejo de conflictos: a) centrarse en la otra persona, el cual corresponde al estilo de ceder o complacer a la contraparte, b) centrarse en uno mismo, el cual corresponde a un estilo orientado a la dominación sobre la otra persona y c) centrarse en el problema, el cual corresponde a un estilo de cooperar o colaborar con la otra parte (Ross \& DeWine, 1988; Kimsey \& Fuller, 2003).

En este marco, el presente estudio se propuso realizar una adaptación del Inventario de Rahim sobre estilos de manejo de conflictos
(Rahim Conflict Organizational Inventory II, ROCI-II Form C; Rahim, 1983, 2001) al contexto de los conflictos entre pares adolescentes compañeros de aula en el bachillerato analizando las propiedades psicométricas de esta versión adaptada.

El interés por incorporar el modelo de Rahim $(1983,2001)$ al estudio de los conflictos entre pares adolescentes en el contexto escolar deriva de la necesidad de explorar otras posibles formas de configuración de los estilos de manejo de conflictos en adolescentes (Luna-Bernal, 2017). A su vez, este modelo contempla, en principio, cinco estilos de manejo de conflictos en lugar de solo tres como los anteriormente usados en este ámbito.

A pesar de que el ROCI-II fue diseñado originalmente para evaluar estilos de manejo de conflictos en el ámbito organizacional, también ha sido empleado en distintos contextos relacionales como los conflictos de pareja, con padres y con amigos (Castellano, Velotti, Crowell, \& Zavattini, 2014; Lin, Lin, Huang, \& Chen, 2016; Ricco \& Sierra, 2017; Zwahr-Castro \& Dicke-Bohmann, 2014), entre otros.

Así mismo, el modelo de Rahim y su cuestionario ROCI-II ya han sido utilizados anteriormente para evaluar estilos de manejo de conflictos entre adolescentes en el contexto escolar. Tal es el caso de los estudios realizados por Chang y Zelihic (2013) en Taiwán, Colsman y Wulfert (2002) en Estados Unidos y De Conti (2014) en Italia.

\section{Estilos de manejo de conflictos en el modelo de Rahim}

Rahim (1983, 2001) estableció los estilos de manejo de conflictos a partir de la interacción de dos dimensiones: la preocupación por sí mismo (concern for self) y la preocupación por otros 
(concern for others). Estas dimensiones representan las orientaciones motivacionales que se le presentan a un sujeto concreto en el curso de un conflicto y su combinación tiene por resultado cinco estilos de manejo de conflictos: a) integrativo (integrating), que indica una alta preocupación tanto por uno mismo como por la otra parte involucrada en el conflicto, el individuo busca una solución en la que se integren las necesidades de ambas partes; b) complaciente o servicial (obliging), que indica una baja preocupación por uno mismo y una alta preocupación por el otro, el sujeto busca complacer a la contraparte; c) dominante (dominating), que indica una alta preocupación por uno mismo y una baja preocupación por la otra parte involucrada en el conflicto, el individuo intenta satisfacer sus propios intereses aun a costa de los demás; d) evitativo (avoiding), que indica una baja preocupación tanto por uno mismo como por la otra parte, el sujeto evita o evade la situación de conflicto; y e) comprometido o transigente (compromising), que indica una preocupación moderada tanto por sí mismo como por la otra parte, el individuo busca llegar a una solución acordada mediante concesiones mutuas.

Para evaluar estos cinco estilos de manejo de conflictos, Rahim (1983) desarrolló el cuestionario ROCI-II. Este instrumento tiene tres versiones (A, B y $\mathrm{C}$ ), que evalúan la manera en que un miembro de una organización maneja sus conflictos con superiores, subordinados y pares, respectivamente. Trabajando con grupos de estudiantes universitarios de pregrado y posgrado, así como con profesores y directivos, Rahim (1983, 2001) y sus colaboradores desarrollaron, corrigieron y seleccionaron un conjunto de reactivos para incluirlos en el instrumento. Dichos reactivos fueron sometidos a seis estudios sucesivos de análisis factorial a fin de seleccionar aquellos que tuvieran cargas factoriales más altas (arriba de .40) y una interpretación congruente con el mode- lo teórico. Así fueron considerados 105 reactivos. Los 28 reactivos, que pasaron a formar parte de la versión final del ROCI II, fueron seleccionados de un conjunto de 35 a partir de un análisis factorial realizado con una muestra nacional de 1.219 directivos en Estados Unidos (Rahim, 1983, 2001; Rahim \& Magner, 1995).

Entre los estudios que han adaptado y validado el ROCI-II en diferentes idiomas y contextos socio-culturales, cabe mencionar los de Dixit y Mallik (2008), Bowles (2009), Munduate, Ganaza y Alcaide (1993) y Ramírez-Landaeta y Borges-Grün (2011), quienes realizaron estudios de validación para India, Australia, España y Brasil, respectivamente. Cabe mencionar que, hasta el momento, los autores no hemos encontrado ningún estudio de validación realizado en población mexicana.

En los estudios señalados se ha replicado la estructura factorial original de cinco factores del ROCI-II, correspondientes a los cinco estilos de manejo de conflictos del modelo teórico de Rahim (1983, 2001). Sin embargo, en otros estudios se ha encontrado un menor número de factores. Al respecto, cabe mencionar el estudio de Hammock, Richardson, Pilkington y Utley (1990), en el que los autores sugieren que la solución de cuatro factores (en lugar de la de cinco) sería más apropiada para el ámbito de los conflictos en las relaciones sociales (padres, amigos y hermanos) que la solución de cinco factores planteada originalmente por Rahim (1983) para los conflictos en las organizaciones. Así mismo, cabe mencionar el estudio de DeBates (1999), que trabajó con una adaptación del ROCI-II para evaluar los estilos de manejo de conflictos que los adolescentes manifiestan cuando tienen conflictos con sus mejores amigos del mismo sexo. El estudio se llevó a cabo con una muestra de 393 adolescentes de 13 a 15 años en Dakota, Estados Unidos. Una vez realizado un análisis factorial exploratorio, la au- 
tora encontró una composición de cuatro factores (compromising/collaborating, accomodating, avoiding, y dominating),y concluyó en la posible presencia de un factor común subyacente a los reactivos correspondientes a los estilos comprometido (compromising) e integrativo (collaborating).

A partir de estos antecedentes, se puede apreciar que, es necesario proseguir con la realización de estudios que analicen la estructura factorial del ROCI-II en diversos contextos. En ese marco, se planteó realizar el presente estudio en una muestra de adolescentes mexicanos estudiantes de bachillerato, considerando el creciente interés que existe actualmente por generar más conocimiento acerca de las maneras en que los adolescentes manejan sus conflictos interpersonales cotidianos (Fahimi \& Tarkhan, 2016; Luna-Bernal \& De Gante-Casas, 2017; Pérez-Archundia \& Gutiérrez-Méndez, 2016).

\section{Estudios que emplean el ROCI-II en muestras de adolescentes}

Los estudios que han empleado el modelo de Rahim (1983, 2001) para evaluar los estilos de manejo de conflictos de los adolescentes en el contexto escolar son escasos (Chang \& Zelihic, 2013; Colsman \& Wulfer, 2002; De Conti, 2014; Luna-Bernal, 2017), y ninguno presenta un análisis de la estructura factorial del instrumento. No obstante, a continuación se hará referencia a dichos trabajos con el fin de ilustrar la manera en que se ha adaptado el ROCI-II a este contexto en particular.

El objetivo del estudio de Chang y Zelihic (2013) fue analizar los estilos de manejo de conflictos que los adolescentes emplean en los conflictos que se les presentan con sus compañeros de clase, así como el impacto de dichos estilos sobre la atmósfera de grupo. Para ello, los autores adaptaron los reactivos del ROCI-II para que en el contenido de estos se hiciera referencia a los compañeros de grupo. Por ejemplo, el reactivo $\mathrm{N}^{\mathrm{o}}$ 2 del cuestionario original de Rahim dice I generally try to satisfy the needs of my peers; pero en la adaptación de Chang y Zelihic (2013) quedó como sigue: I generally try to satisfy the needs of my classmates or friends (p. 1240), y así sucesivamente. Con este cuestionario adaptado, los autores evaluaron los cinco estilos de manejo de conflicto del modelo de Rahim. La muestra del estudio estuvo conformada por 843 adolescentes, con edades de entre 15 y 17 años, los cuales pertenecían a 16 bachilleratos (high schools) distribuidos en el norte, centro, sur y este de Taiwán.

Por su parte, De Conti (2014) en Italia se planteó evaluar el impacto que pueden tener los programas de entrenamiento en debate competitivo sobre los estilos de manejo de conflictos de los adolescentes. Para ello, conformó un grupo experimental con 42 estudiantes de bachillerato con edades de entre 16 y 19 años que participaban en un programa de entrenamiento en debate competitivo en la Universidad de Padua y un grupo de control compuesto por 87 estudiantes que no participaban en dicho programa. De acuerdo con De Conti (2014), si bien el ROCI- II ha sido diseñado originalmente considerando el contexto organizacional, es posible contextualizar cada reactivo de acuerdo con los tipos de relaciones experimentadas por los sujetos, incluyendo sus relaciones con compañeros de escuela, profesores o padres. De acuerdo con los intereses de estudio, "la elección estuvo orientada a los conflictos con compañeros de aula (classmates)" (p. 127).

Por otro lado, Colsman y Wulfer (2002) realizaron un estudio con 61 estudiantes de una preparatoria pública ubicada en el estado de Nueva York, Estados Unidos, con una media de edad de 15.9 años. El objetivo de su estudio fue ana- 
lizar la posible función de los estilos de manejo de conflictos como indicadores de problemas de conducta en adolescentes (particularmente, del consumo de sustancias psicoactivas). Según informan los autores, con base en estudios previos, el cuestionario ROCI-II fue adaptado para los adolescentes considerando cuatro estilos de manejo de conflictos (en vez de cinco): a) evitar (avoidance), b) contender (contentiousness), c) ceder (accomodation) y d) cooperar (cooperation). Este último estilo combinaba, en un mismo factor, las escalas de integración (integration) y compromiso (compromise) del inventario original. Los autores también indican que en un estudio previo Weider-Hatfield (1988) reportó una confiabilidad alfa de Cronbach de .95 para esta escala combinada.

Por su parte, en México, Luna-Bernal (2017) realizó un estudio con una muestra de 194 estudiantes de bachillerato con edades de entre 15 y 18 años, el cual tenía por objetivo evaluar la relación entre los estilos de manejo de conflictos y la empatía multidimensional. El autor utilizó el ROCI-II en una versión adaptada a los conflictos entre pares adolescentes en el aula. Para realizar la adaptación se utilizó un procedimiento semejante al usado por Chang y Zelihic (2013), es decir, se adecuó la redacción de cada uno de los reactivos al contexto de las relaciones de los adolescentes con sus compañeros de aula. Por ejemplo, el reactivo $\mathrm{N}^{\mathrm{o}} 2$ originalmente era Ante un problema de trabajo, generalmente trato de satisfacer los deseos de mi compañero, mientras que el reactivo adaptado quedó como Ante un problema, generalmente trato de satisfacer los deseos de mi compañero(a).

Como se puede observar, en los estudios mencionados se ha contemplado una composición factorial distinta del ROCI-II. Mientras que Chang y Zelihic (2013), De Conti (2014) y Luna-Bernal (2017) emplearon un modelo de cinco estilos; Colsman y Wulfert (2002) consideraron solo cuatro. De manera similar, como ya se ha señalado, estudios realizados en otros contextos han identificado una estructura pentafactorial del ROCI-II (Bowles, 2009; Dixit \& Mallik , 2008; Munduate et al., 1993; Ramírez-Landaeta \& Borges-Grün, 2011), mientras que otros han hallado elementos a favor de una estructura de cuatro factores (DeBates, 1999; Hammock et al., 1990). A raíz de lo mencionado, uno de los objetivos que se planteó el presente estudio fue analizar la estructura factorial del ROCI-II en una muestra de adolescentes mexicanos de bachillerato, con el fin de contribuir a esta discusión sobre la estructura factorial del instrumento.

\section{El modelo de Ross y DeWine}

En diversos estudios anteriores, el Cuestionario sobre Estilos de Mensajes en el Manejo de Conflictos (Ross-DeWine Conflict Management Message Style Instrument, CMMS) de Ross y DeWine (1988) ha mostrado ser consistente y útil para conceptualizar y evaluar estilos de manejo de conflictos en muestras de adolescentes mexicanos, tanto en el contexto de los conflictos parento-filiales (Luna-Bernal, 2014; Luna-Bernal, Laca-Arocena, \& Cedillo-Navarro, 2012), como en los conflictos entre adolescentes en el contexto escolar (Luna \& Laca, 2014; Luna-Bernal \& De Gante-Casas, 2017). En este trabajo se analizan las posibles relaciones entre los estilos del modelo de Rahim $(1983,2001)$ y los de Ross y DeWine (1988) con el fin de añadir al presente análisis un criterio de validez convergente.

Ross y DeWine (1988) propusieron tres estilos de manejo de conflictos evaluados con el CMMS: a) el estilo enfocado en uno mismo (concern for self), en el que el individuo se concentra en su propio interés personal, sin considerar el 
interés de la otra persona, adoptando actitudes y comportamientos dominantes; b) el estilo enfocado en la otra parte (concern for other), en el que el individuo deja de lado los intereses propios y se centra en complacer a la otra persona; por último, c) el estilo enfocado en el problema (concern for issue), en el que el sujeto decide invitar a la otra parte a concentrarse en el problema que es materia de discusión, así como a colaborar o a convenir en alguna solución que satisfaga, al menos parcialmente, los intereses de ambos.

\section{Objetivos}

En el marco de todo lo anteriormente expuesto, el presente trabajo se planteó como objetivos específicos los siguientes: a) analizar la estructura factorial del Inventario de Rahim (ROCI-II forma $\mathrm{C}$ ) en su versión adaptada al contexto de los conflictos entre pares en el aula en una muestra de adolescentes estudiantes de bachillerato con el fin de obtener y ponderar información relativa a su posible estructura pentafactorial o tetrafactorial, así como de identificar sus características de validez y confiabilidad; b) analizar posibles diferencias significativas por sexo, edad y grado escolar en la muestra de estudio en relación con los estilos de manejo de conflictos del modelo de Rahim; y c) analizar las posibles relaciones entre las escalas del Inventario de Rahim y las del CMMS, con el fin de obtener información sobre la validez convergente.

\section{Método \\ Participantes}

La muestra estuvo compuesta por 663 estudiantes de bachillerato con rango de edad de entre 15 y 19 años $(M=16.67$; $\mathrm{DE}=1.01)$. Los participantes pertenecían a una escuela preparatoria pública ubicada en la Zona Metropolitana de Guadalajara, Jalisco, México, y se encontraban distribuidos en los seis grados (semestres) que comprende el bachillerato (Educación Media Superior) en México.

Para los fines del análisis de la variable de edad se formaron dos grupos: a) 15 y 16 años y b) 17 a 19 años. Así mismo, para facilitar una comparación más clara de los grupos, se procedió a evaluar la variable de grado escolar por anualidades, considerando tres niveles: primer año (semestres 1 y 2), segundo año (semestres 3 y 4) y tercer año (semestres 5 y 6). En la Tabla 1 se puede observar la distribución por sexo según edad y grado escolar.

Tabla 1. Distribución de la muestra en edad y grado, en función del sexo $(\mathrm{N}=663)$.

\begin{tabular}{lccc}
\hline & Mujeres & Hombres & Total \\
\hline 15 y 16 años & $165(24.9 \%)$ & $142(21.4 \%)$ & $307(46.3 \%)$ \\
17 a 19 años & $182(27.5 \%)$ & $174(26.2 \%)$ & $356(53.7 \%)$ \\
Primer año & $153(23.1 \%)$ & $146(22.0 \%)$ & $299(45.1 \%)$ \\
Segundo año & $112(16.9 \%)$ & $102(15.4 \%)$ & $214(32.3 \%)$ \\
Tercer año & $82(12.4 \%)$ & $68(10.3 \%)$ & $150(22.6 \%)$ \\
Total & $347(52.3 \%)$ & $316(47.7 \%)$ & $663(100 \%)$ \\
\hline \multicolumn{5}{l}{ Nota. Los porcentajes son en relación con el total de la muestra. }
\end{tabular}

Instrumentos

Inventario de Rahim sobre Estilos de Manejo de Conflictos(Rahim Organizational Conflict Inventory II, form C; ROCI-II forma C; Rahim, 1983). Este instrumento informa sobre la frecuencia con que los participantes perciben utilizar los estilos de manejo de conflictos, según el modelo de Rahim (1983, 2001). En el presente estudio se tomó como punto de partida la versión C en la traducción castellana del instrumento realizada por Munduate et al. (1993). Para realizar 
la adaptación se utilizó un procedimiento similar al empleado por Chang y Zelihic (2013) y Luna-Bernal (2017), que consistió en la adecuación de cada uno de los 28 reactivos originales al contexto de las relaciones de los adolescentes con sus compañeros de aula. Por ejemplo, Ante una dificultad con mi compañero(a), intento analizar la situación con él o ella para encontrar una solución aceptable para ambos (reactivo $\mathrm{N}^{\mathrm{o}} 1$ ), Ante un problema, generalmente trato de satisfacer los deseos de mi compañero(a) (reactivo $\mathrm{N}^{\mathrm{o}}$ 2). El formato de respuesta es una escala Likert de cinco puntos que va desde $1=$ Nunca hasta 5 = Siempre. Los índices de confiabilidad alfa de Cronbach reportados por Munduate et al. (1993) fueron de $.77, .76, .75, .70$ y .62 para los estilos integrativo, complaciente, dominante, evitativo y comprometido, respectivamente.

Cuestionario sobre Estilos de Mensajes en el Manejo de Conflictos (Ross-DeWine Conflict Management Message Style Instrument; CMMS). Se trata de un cuestionario de autoinforme que evalúa los estilos de manejo de conflictos de acuerdo con el modelo de Ross y DeWine (1988). Cada reactivo representa un mensaje dado por un sujeto a su contraparte en una situación de conflicto, por ejemplo: ¿Cómo puedo hacerte sentir bien otra vez? (reactivo $\mathrm{N}^{\mathrm{o}} 2$ ), Estoy muy molesto por algunas cosas que están pasando; ¿podemos hablar sobre ellas? (reactivo $\mathrm{N}^{\mathrm{o}} 3$ ). El formato de respuesta es una escala Likert que va desde $1=$ Nunca digo cosas como esto hasta $5=$ Generalmente digo cosas como esto.

El CMMS fue elaborado originalmente por Ross y DeWine (1988). Mejía-Ceballos y Laca-Arocena (2006), y Laca, Mejía y Mayoral (2011) llevaron a cabo la traducción y validación a la lengua castellana. Luna y Laca (2014), por su parte, realizaron un nuevo estudio de validación con una muestra $(\mathrm{N}=1074)$ de estudiantes mexicanos de secundaria, bachillerato y licenciatura con un rango de edad de entre 11 y 25 años. En dicho estudio, el instrumento constó de 13 reactivos divididos en tres escalas: a) estilo enfocado en uno mismo, b) estilo enfocado en la otra parte y c) estilo enfocado en el problema, con índices de confiabilidad alfa de Cronbach de $.68, .72$ y .83 , respectivamente.

En el presente estudio, los participantes respondieron a los 18 reactivos del CMMS original; no obstante, para la calificación se consideraron solamente los 13 reactivos resultantes en la composición factorial revelada por el estudio de Luna y Laca (2014), debido a que éste constituye el estudio de validación de referencia para muestras del grupo etario al que se orienta la presente investigación.

\section{Procedimiento}

Se obtuvo la colaboración en el proyecto de las autoridades escolares y se acordó un horario para la aplicación de los instrumentos. Los investigadores, acompañados de tres auxiliares previamente capacitados, acudieron a las aulas de los grupos seleccionados y solicitaron a los estudiantes su participación voluntaria y anónima, para lo cual se les informó sobre los objetivos de la investigación. Por su parte, aquellos alumnos que no desearan intervenir fueron invitados a retirarse del aula. A los participantes se les explicó que las respuestas a este tipo de instrumentos no son correctas ni incorrectas y se los invitó a contestar con sinceridad. Así mismo, se les garantizó el manejo estrictamente confidencial y estadístico de la información y su uso para fines exclusivamente científicos. 


\section{Consideraciones éticas}

La presente investigación es considerada de bajo riesgo conforme a la Ley General de Salud vigente en los Estados Unidos Mexicanos $(\mathrm{H}$. Congreso de la Unión, 1984); y se llevó a cabo en adhesión a las especificaciones del Código Ético del Psicólogo de la Sociedad Mexicana de Psicología (2010), así como a los principios éticos y código de conducta de la American Psychological Association (2017).

\section{Análisis estadístico}

Se llevó a cabo un análisis factorial confirmatorio empleando el método de mínimos cuadrados generalizados (GLS). Con base en la literatura y el marco teórico, se especificó inicialmente un modelo de cinco factores (variables latentes). Se tomó como base la distribución de los 28 reactivos del inventario original de Rahim $(1983,2001)$, los cuales figuraron como indicadores (variables observadas). Siguiendo la recomendación de diversos autores (Byrne, 2010; Ferrando \& Anguiano-Carrasco, 2010; Hair, Anderson, Tatham, \& Black, 1999) relativa a utilizar varias medidas para evaluar la bondad del ajuste, en el presente trabajo se utilizaron las siguientes: el cociente entre Chi cuadrada y sus grados de libertad $(\chi 2 / \mathrm{gl})$, el residuo cuadrático medio de aproximación (RMSEA), el índice de bondad de ajuste (GFI), el índice de bondad de ajuste en su modalidad corregida (AGFI) y el índice de bondad de ajuste de parsimonia (PGFI).

Generalmente, se considera un ajuste adecuado cuando $\chi 2 / \mathrm{gl}$ es menor a 3 (Bentler \& Bonett, 1980; Ruiz, Pardo, \& San Martín, 2010). Valores RMSEA menores a .05 se consideran excelentes, pero entre .05 y .08 indicarían un ajuste admisible (Ferrando \& Anguiano-Carrasco,
2010). Los índices GFI y AGFI cercanos a 1 indican excelente ajuste (Byrne, 2010). Por su parte, las magnitudes de PGFI situadas entre $.05 \mathrm{y}$ .07 se consideran aceptables (Escobedo-Portillo, Hernández-Gómez, Estebané-Ortega, \& Martínez-Moreno, 2016).

Con el fin de identificar posibles efectos del sexo, edad o grado escolar en las variables de estudio, se llevó a cabo un análisis multivariado de la varianza (MANOVA) trifactorial con diseño de 2 × 2 × 3 (dos niveles de sexo por dos de edad, por tres de grado escolar).

Finalmente, con el objetivo de explorar las relaciones entre los estilos de gestión de conflictos del modelo de Rahim $(1983,2001)$ y los del modelo de Ross y DeWine (1988), se realizó un análisis de correlación de Pearson entre las escalas de ambos instrumentos. De acuerdo con Cohen (como se citó en Coolican, 2005), los valores de $r$ cercanos a $.1, .3$ y .5 se consideran correlaciones débiles, moderadas y fuertes, respectivamente.

Todos los cálculos se llevaron a cabo utilizando los programas estadísticos SPSS 21 (IBM Corporation, 2012) y AMOS 21 (Arbuckle, 2012).

\section{Resultados}

Se llevó a cabo un primer análisis factorial confirmatorio (AFC) en el que se consideraba el modelo original de cinco factores del Inventario de Rahim. Todas las cargas factoriales oscilaron entre .50 y .75 . Por su parte, los coeficientes de determinación estandarizados (varianza explicada) fueron aceptables, oscilando entre .25 y .56 . Por su parte, algunos de los índices de bondad de ajuste considerados resultaron adecuados, pudiendo mantenerse la hipótesis de bondad de ajuste $(\chi 2 / \mathrm{gl}=2.697, p<.001 ; \mathrm{RMSEA}=.051)$, 
mientras que otros solo indicaron un ajuste moderado $(\mathrm{GFI}=.901 ; \mathrm{AGFI}=.882 ; \mathrm{PGFI}=$.755). Cabe destacar que, no obstante lo anterior, se observó una alta correlación entre los factores comprometido e integrativo $(r=.92)$, al revisar las correlaciones entre los factores del modelo.

Dada la fuerte correlación hallada entre los factores integrativo y comprometido del modelo original, se decidió llevar a cabo un segundo AFC con un modelo revisado, en atención a los estudios previos que han considerado una posible estructura de cuatro factores del Inventario de Rahim (Colsman \& Wulfert, 2002; DeBates, 1999; Hammock et al., 1990). Dichos estudios han considerado las escalas de los estilos integrativo y comprometido como integradas en un solo factor.

Como puede observarse en la Figura 1, para la especificación de este modelo revisado, se integraron los factores integrativo y comprometido del modelo original en un solo factor denominado estilo cooperativo, al cual se asociaron como indicadores los mismos reactivos de aque1los dos factores.

Los resultados de este segundo AFC se presentan en la Figura 1. Como puede observarse, todas las cargas factoriales fueron de moderadas a altas, oscilando entre .48 y .74. Los coeficientes de determinación estandarizados $(r 2)$ fueron aceptables (superiores al 20\% de la varianza), oscilando entre .23 y .56 . Por su parte, algunos de los índices de bondad de ajuste considerados resultaron adecuados, lo que sostiene la hipótesis de bondad de ajuste $(\chi 2 / \mathrm{gl}=2.697, p<.001$; RMSEA $=.051)$; mientras que otros indicaron un ajuste moderado $(\mathrm{GFI}=.900 ; \mathrm{AGFI}=.882$;

PGFI $=$.762).

Considerando esta solución factorial, se calcularon para cada participante cuatro puntuaciones: a) estilo dominante, b) estilo complaciente, c) estilo evitativo y d) estilo cooperativo. Los índices de confiabilidad alfa de Cronbach co- rrespondientes a cada una de estas cuatro escalas fueron de .70, .79, .72 y .88, respectivamente. Las puntuaciones medias obtenidas por los participantes en cada una de las escalas mencionada fueron de $2.83(\mathrm{DE}=.81), 2.28(\mathrm{DE}=.77), 3.02$ $(\mathrm{DE}=.81)$ y $3.21(\mathrm{DE}=.79)$, respectivamente.

A continuación se llevó a cabo el análisis MANOVA para evaluar posibles diferencias por sexo, edad y grado escolar. No resultaron significativos los efectos de interacción, grado ni edad. Únicamente resultaron estadísticamente significativos los efectos principales de la variable de sexo (Lambda de Wilks $(4 / 650)=.943, p<.001)$. En la Tabla 2 se presentan las medias, las desviaciones estándar y los valores de $\mathrm{F}$ y $p$ correspondientes. Como se puede observar, en el estilo dominante los varones puntuaron, de manera estadísticamente significativa, más alto que las mujeres, mientras que estas puntuaron más alto en el estilo cooperativo.

Tabla 2. Diferencias en función del sexo en estilos de manejo de conflictos $(\mathrm{N}=663)$.

\begin{tabular}{lccc}
\hline & $\begin{array}{c}\text { Mujeres } \\
(\mathbf{n}=\mathbf{3 4 7}) \\
\mathbf{M}(\mathbf{D E})\end{array}$ & $\begin{array}{c}\text { Hombres } \\
(\mathbf{n}=\mathbf{3 1 6})\end{array}$ & $\mathbf{F}(\mathbf{1} / \mathbf{6 6 1})$ \\
\hline Dominante & $2.72(.79)$ & $2.96(.81)$ & $14.771^{* * *}$ \\
Complaciente & $2.25(.78)$ & $2.31(.75)$ & .969 \\
Evitativo & $3.06(.81)$ & $2.97(.80)$ & 1.863 \\
Cooperativo & $3.27(.78)$ & $3.14(.81)$ & $4.833^{*}$ \\
\hline Nota. ${ }^{*} p .05,{ }^{* * *} p<.001$ & &
\end{tabular}

Finalmente, con el objetivo de explorar las relaciones entre los estilos de gestión de conflictos del modelo de Rahim $(1983,2001)$ y los del modelo de Ross y DeWine (1988), se llevó a cabo un análisis de correlación de Pearson entre las escalas de ambos instrumentos. A raíz del mencionado efecto del sexo sobre las variables de estudio, se decidió emplear el análisis de cor- 


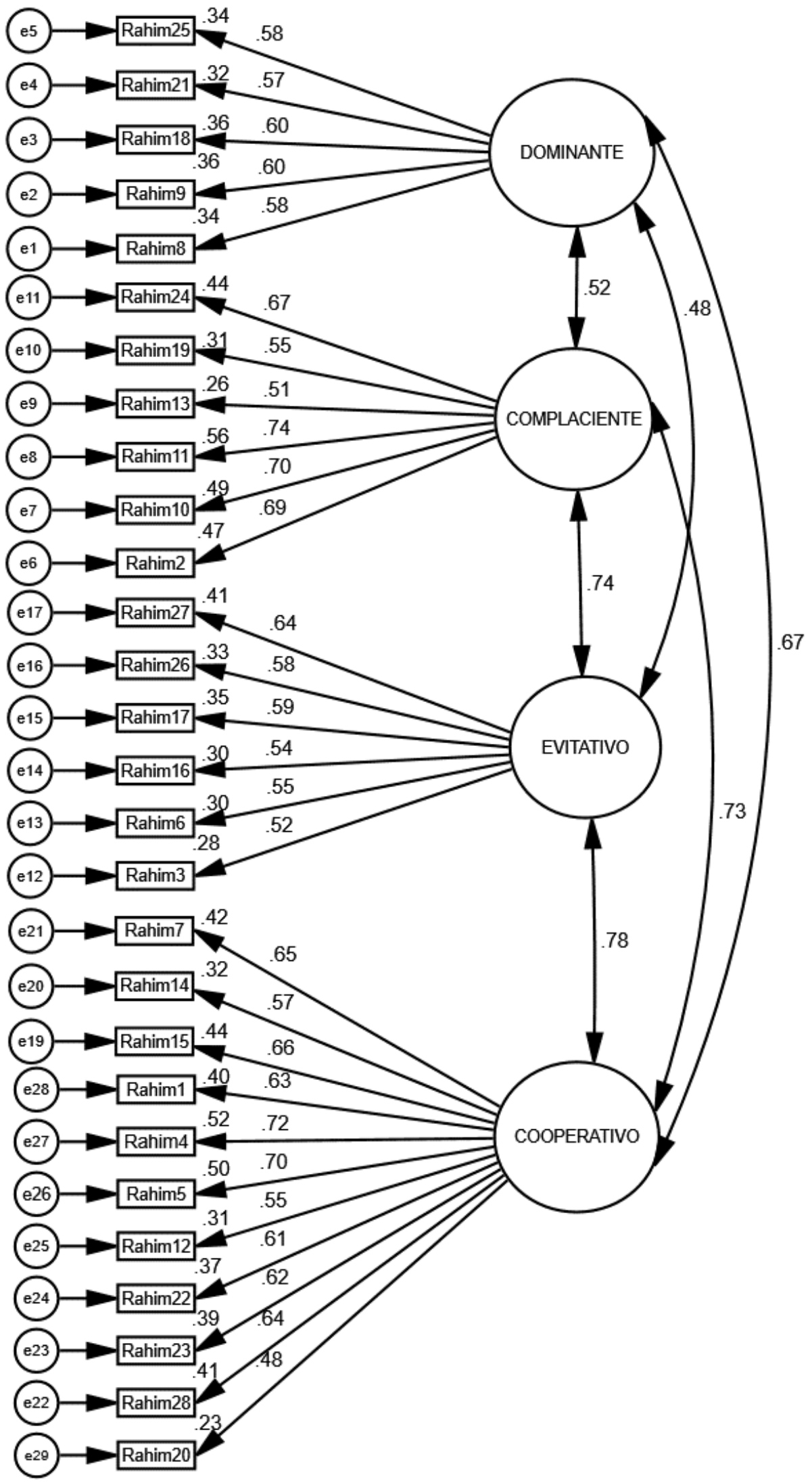

Figura 1

Análisis factorial confirmatorio del Inventario de Rahim adaptado. Modelo revisado. 
relación parcial para controlar esta variables. Los resultados se muestran en la Tabla 3 . Como se puede observar, tanto el estilo dominante como el complaciente correlacionaron positivamente con todos los estilos del modelo de Ross y DeWine (1988), de manera estadísticamente significativa. Por su parte, los estilos evitativo y cooperativo correlacionaron positivamente con los estilos centrado en la otra parte y centrado en el problema, de manera estadísticamente significativa.

Tabla 3. Coeficientes de correlación parcial entre estilos de manejo de conflictos con control de la variable sexo $(\mathrm{N}=$ $660)$.

\begin{tabular}{lccc}
\hline & $\begin{array}{c}\text { Centrado } \\
\text { en uno } \\
\text { mismo }\end{array}$ & $\begin{array}{c}\text { Centrado } \\
\text { en la otra } \\
\text { parte }\end{array}$ & $\begin{array}{c}\text { Centrado en } \\
\text { el problema }\end{array}$ \\
\hline Dominante & $.19 * * *$ & $.30 * * *$ & $.33 * * *$ \\
Complaciente & $.23 * * *$ & $.40 * * *$ & $.34 * * *$ \\
Evitativo & -.04 & $.28 * * *$ & $.31 * * *$ \\
Cooperativo & -.05 & $.34 * * *$ & $.52 * * *$ \\
\hline Nota. ${ }^{* * *} p<.001$. & & &
\end{tabular}

\section{Discusión}

El presente trabajo planteó como objetivos específicos los siguientes: a) analizar la estructura factorial de la versión adaptada del Inventario de Rahim (ROCI-II forma C) en una muestra de adolescentes bachilleres, identificando sus propiedades psicométricas de validez y confiabilidad; b) analizar posibles diferencias significativas de acuerdo al sexo, edad y grado escolar en la muestra de estudio, con relación a los estilos de manejo de conflictos del modelo de Rahim; y c) analizar las posibles relaciones entre las escalas del Inventario de Rahim y las del CMMS de Ross y DeWine (1988) a fin de obtener mayor información sobre la validez convergente, ya que este último instrumento ha sido, hasta el momento, el más empleado con muestras de adolescentes mexicanos.

Con respecto al primer objetivo, los resultados sugieren, en ambos modelos, un grado de ajuste similar al encontrado por Rahim en sus estudios de validación del ROCI-II, en los que los valores de GFI y AGFI oscilaron de .82 a .89 y de .78 a.87, respectivamente (Rahim, 2001; Rahim \& Magner, 1995). Este resultado fue calificado como "un moderado ajuste a los datos" por el propio autor (Rahim \& Magner, 1995, p. 125).

No obstante, el presente estudio el modelo pentafactorial evidenció la dificultad de una alta correlación entre los factores integrativo y comprometido, lo cual es un argumento a favor de la estructura tetrafactorial del instrumento. Como se señaló, este hallazgo es congruente con estudios previos que han considerado una posible estructura de cuatro factores del Inventario de Rahim en el contexto de las relaciones sociales (DeBates, 1999; Hammock et al., 1990; Colsman \& Wulfert, 2002). Por lo dicho, es posible concluir que la presente investigación aporta información relevante para sustentar que el Inventario de Rahim adaptado para evaluar estilos de manejo de conflictos entre pares adolescentes en el contexto escolar del bachillerato, probablemente, descanse sobre una estructura conformada por cuatro factores: a) estilo dominante, b) estilo complaciente, c) estilo evitativo y d) estilo cooperativo. Los índices de confiabilidad alfa de Cronbach resultaron aceptables para los primeros tres estilos, ya que fueron superiores a .70; y resultó un índice bueno para el estilo cooperativo, ya que se ubicó arriba de .80 (Frías-Navarro, 2014).

Con relación al segundo objetivo, como se pudo observar, no se encontraron diferencias significativas por edad ni grado escolar en ninguno de los estilos de manejo de conflictos. A este respecto, Luna y Laca (2014) han hecho notar que las diferencias evolutivas en estas variables son 
más visibles en investigaciones que toman rangos de edad más amplios (desde la preadolescencia a la juventud o adultez, por ejemplo). Por esto, en estudios como el presente, que toma un rango relativamente corto, de 15 a 19 años, no es inusual que las pruebas no logren identificar una diferencia significativa por grupos de edad. Un razonamiento similar podría aplicarse al grado escolar, tomando en cuenta la evidencia proporcionada por estudios que han encontrado diferencias significativas pero solo al comparar grupos de participantes de diferentes niveles educativos, por ejemplo, entre secundaria, bachillerato y licenciatura (Markovits \& St-Onge, 2009; Saville \& Swoap, 2006).

En relación con las diferencias de sexo, en el presente estudio los hombres puntuaron más alto que las mujeres en el estilo dominante, mientras que ellas puntuaron más alto en el estilo cooperativo. Este hallazgo es congruente con la literatura, en la que se ha encontrado de manera consistente una inclinación de las mujeres hacia los estilos cooperativos y constructivos, y de los varones hacia los estilos competitivos y agresivos (Alexander, 2000; Garaigordobil, 2009, 2012; Garaigordobil \& Maganto, 2011; Garaigordobil et al., 2016; Laca et al., 2006; Luna \& Laca, 2014). La explicación más aceptada de estos resultados sostiene que esto podría deberse a que los hombres y las mujeres adolescentes son sujetos de procesos de socialización diferencial, en el sentido de los roles de género tradicionales, de modo que en ellas se habrían conformado características de corte más expresivo y relacional y en ellos, características más de tipo agresivo e instrumental (Rebollo-Catalán, Ruiz-Pinto, \& García-Pérez, 2017; Rocha-Sánchez, 2008).

Con respecto al tercer objetivo, tanto el estilo dominante como el complaciente correlacionaron positivamente con todos los estilos del modelo de Ross y DeWine (1988), mientras que los estilos evitativo y cooperativo correlacionaron positivamente con los estilos centrado en la otra parte y centrado en el problema. Las correlaciones tendieron a ser de grado moderado (Cohen, 1988; Coolican, 2005), lo que podría indicar que ambos instrumentos evalúan constructos distintos aunque relacionados. En relación con esto, es interesante subrayar que el propio Rahim (2001) señalaba que la principal evidencia para los modelos de tres estilos proviene de la literatura sobre comunicación; mientras que la construcción del instrumento de Ross y DeWine (1988) se basa, precisamente, en un enfoque comunicativo. Sin embargo, las correlaciones encontradas entre ambos instrumentos proporcionan datos a favor de la validez convergente del Inventario de Rahim adaptado, que se ha empleado en el

presente estudio.

En conclusión, los hallazgos del presente estudio aportan información relevante acerca de la validez y confiabilidad del Inventario de Rahim adaptado al contexto de los conflictos que se les presentan a los adolescentes con sus compañeros de aula en el bachillerato. Como se señaló, los estudios desarrollados anteriormente en el ámbito iberoamericano sobre estilos de manejo de conflictos en adolescentes en contexto escolar han estado basados en el modelo de tres estilos (colaborar, competir y ceder). La incorporación del modelo de Rahim en esta área de investigación permitirá ampliar los análisis para explorar otras posibles formas de configuración de los estilos de manejo de conflictos en los adolescentes.

Dentro de las limitaciones del presente estudio, se puede mencionar que el rango de edad estuvo circunscrito a la adolescencia media y tardía (de 15 a 19 años), por lo que se aconseja emplear en estudios posteriores muestras con un rango de edad más amplio, a efectos de posibilitar comparaciones entre diversos grupos etarios. También se recomienda la incorporación de otras 
variables de relevancia teórica y práctica, tales como las relativas a competencias y habilidades (p. ej., toma de decisiones, autorregulación emocional, toma de perspectiva, comunicación interpersonal, argumentación, entre otras) a fin de evaluar su relación con los estilos de manejo de conflictos del modelo de Rahim.

\section{Referencias}

Abas, N. A. H. (2010). Emotional intelligence and conflict management styles (Tesis de maestría). Recuperado de http://www2.uwstout.edu/content/lib/thesis/2010/2010abasn.pdf

Alexander, K. L. (2000). Prosocial behaviors of adolescents in work and family life: Empathy and conflict resolution strategies with parents and peers (Tesis doctoral). Recuperado de https://etd.ohiolink.edu/ pg_1?5612014979328

American Psychological Association (2017). Ethical principles of psychologists and code of conduct (2002, as amended 2010, 2016). Recuperado de http://www. apa.org/ethics/code/index.aspx

Arbuckle, J. L. (2012). AMOS (versión 21.0) [Software de computación]. Chicago: IBM SPSS.

Bentler, P. M., \& Bonett, D. G. (1980). Significance tests and goodness of fit in the analysis of covariance structures. Psychological Bulletin, 88(3), 588-606. doi: 10.1037/0033-2909.88.3.588

Blake, R. R., \& Mouton, J. S. (1970). The fifth achievement. Journal of Applied Behavioral Science, 6(4), 413-426. doi: 10.1177/002188637000600403

Bowles, T. (2009). A comparison of two measures of communication and the communication style of university students. Electronic Journal of Applied Psychology: General Articles, 5(1), 53-66. Recuperado de http://pandora.nla.gov.au/tep/51365

Byrne, B. M. (2010). Structural equation modeling with AMOS. Basic concepts, applications, and program- ming ( $2^{\mathrm{a}}$ ed.). New York: Taylor and Francis.

Castellano, R., Velotti, P., Crowell, J. A., \& Zavattini, G. C. (2014). The role of parents' attachment configurations at childbirth on marital satisfaction and conflict strategies. Journal of Child and Family Studies, 23(6), 1011-1026. doi: 10.1007/s10826-013-9757-7

Chang, L. C., \& Zelihic, M. (2013). The study of conflict management among Taiwanese adolescents. Life Science Journal, 10(3), 1231-1241. Recuperado de http://www.lifesciencesite.com/lsj

Cohen, J. (1988). Statistical power analysis for the behavioral sciences (2a ed.). Hillsdale, NJ: Lawrence Erlbaum.

Colsman, M., \& Wulfert, E. (2002). Conflict resolution style as an indicator of adolescents' substance use and other problem behaviors. Addictive Behaviors, 27(4), 633-648. doi: 10.1016/S0306-4603(01)00198-8

Coolican, H. (2005). Métodos de investigación y estadistica en psicología ( $3^{\mathrm{a}}$ ed.; Trads. G. Padilla-Sierra, S. M. Olivares-Bari, \& J. L. Núñez-Herrejón). México: Manual Moderno.

DeBates, D. A. (1999). Adolescents and conflict with peers: Relationships between personality factors and conflict resolution strategies (Tesis doctoral). Recuperado de http://lib.dr.iastate.edu/rtd/12448

De Conti, M. (2014). The impact of competitive debate on managing the conflict communication strategies of Italian students. Argumentation and Advocacy, 51(2), 123-131. doi: 10.1080/00028533.2014.11821843

Dixit, M., \& Mallik, D. (2008). Assessing suitability of Rahim Organizational Conflict Inventory-II in Indian family-owned-and-managed businesses. International Journal of Business Insights \& Transformation, 2(1), 28-38.

Escobedo-Portillo, M. T., Hernández-Gómez, J. A., Estebané-Ortega, V., \& Martínez-Moreno, G. (2016). Modelos de ecuaciones estructurales: Características, fases, construcción, aplicación y resultados. Ciencia y Trabajo, 18(55), 16-22. doi: 10.4067/S0718- 


\section{4}

Fahimi, N., \& Tarkhan, M. (2016). The relationship between conflict resolution strategies and adolescent mental health among female high school students. Journal of Psychology and Behavioral Studies, 4(6), 209-217. Recuperado de http://www.jpbsjournal. com/wp-content/uploads/paper132.pdf

Ferrando, P. J., \& Anguiano-Carrasco, C. (2010). El análisis factorial como técnica de investigación en psicología. Papeles del Psicólogo, 31(1), 18-33.Recuperado de http://www.papelesdelpsicologo.es

Frías-Navarro, D. (2014). Apuntes de SPSS. Universidad de Valencia. Recuperado de https://www.uv.es

Garaigordobil, M. (2009). Evaluación del programa Dando pasos hacia la paz. Informe de investigación. Vitoria-Gasteiz, España: Servicio Central de Publicaciones del Gobierno Vasco. Recuperado de http://www. argia.eus

Garaigordobil, M. (2012). Cooperative conflict-solving during adolescence: Relationships with cognitive-behavioural and predictor variables. Infancia y Aprendizaje, 35(2), 151-165. doi: 10.1174/021037012800217998

Garaigordobil, M., Machimbarrena, J. M., \& Maganto, C. (2016). Adaptación española de un instrumento para evaluar la resolución de conflictos (Conflictalk): Datos psicométricos de fiabilidad y validez. Revista de Psicología Clínica con Niños y Adolescentes, 3(2), 59-67. Recuperado desde http://www.revistapcna. com

Garaigordobil, M., \& Maganto, C. (2011). Empatía y resolución de conflictos durante la infancia y la adolescencia. Revista Latinoamericana de Psicología, 43(2), 255-266. Recuperado de http://www.redalyc. org/revista.oa? $\mathrm{id}=805$

Hair, J., Anderson, R., Tatham, R., \& Black, W. (1999). Análisis multivariante (Trad. E. Prentice \& D. Cano). Madrid: Prentice Hall Iberia.

Hammock, G. S., Richardson, D. R., Pilkington, C. J., \& Utley, M. E. (1990). Measurement of conflict in close interpersonal relationships. Personality and Individual Differences, 11(6), 577-583. doi: 10.1016/01918869(90)90040-X

H. Congreso de la Unión (1984). Ley General de Salud. México: Cámara de Diputados del H. Congreso de la Unión. Recuperado de http://www.diputados.gob. mx/LeyesBiblio/pdf/142_110518.pdf

IBM Corporation (2012). IBM SPSS Statistics for Windows (Versión 21.0) [Software de computación]. Armonk, NY: IBM.

Kimsey, W. D., \& Fuller, R. M. (2003). Conflictalk: An instrument for measuring youth and adolescent management message styles. Conflict Resolution Quarterly, 21(1), 69-78. doi: 10.1002/crq.49

Laca, F., Alzate, R., Sánchez, M., Verdugo, J., \& Guzmán, J. (2006). Communication and conflict in young Mexican students: Messages and attitudes. Conflict Resolution Quarterly, 24(1), 31-54. doi: 10.1002/crq.156

Laca, F. A., Mejía, J. C., \& Mayoral, E. G. (2011). Conflict communication, decision-making, and individualism in Mexican and Spanish university students. Psychology Journal, 8(1), 121-135. Recuperado de https:// www.researchgate.net/publication/233259883

Lin, W. F., Lin, Y. C., Huang, C. L., \& Chen, L. H. (2016). We can make it better: "We" moderates the relationship between a compromising style in interpersonal conflict and well-being. Journal of Happiness Studies, 17(1), 41-57. doi: 10.1007/s10902-014-9582-8

Luna, A. C. A., \& Laca, F. A. (2014). Estilos de mensajes en el manejo de conflictos en adolescentes y jóvenes mexicanos. Boletín de Psicología, 110, 37-51. Recuperado de https://www.uv.es/seoane/boletin/boletin. html

Luna-Bernal, A. C. A. (2014). Efecto de la comunicación parento-filial sobre los estilos personales de manejo de conflictos en adolescentes bachilleres. Uaricha, Revista de Psicología, 11(24), 118-133. Recuperado de http://www.revistauaricha.umich.mx/ojs_uaricha/ index.php/urp/issue/view/16

Luna-Bernal, A. C. A. (2017). Relación entre estilos de 
manejo de conflictos y empatía multidimensional en adolescentes bachilleres. Revista Iberoamericana de las Ciencias Sociales y Humanisticas, 6(12), 80-106. doi: 10.23913/ricsh.v6i12.126

Luna-Bernal, A. C. A., \& De Gante-Casas, A. (2017). Empatía y gestión de conflictos en estudiantes de secundaria y bachillerato. Revista de Educación y Desarrollo, 40, 27-37. Recuperado de http://www.cucs. udg.mx/revistas/edu_desarrollo

Luna-Bernal, A. C. A., Laca-Arocena, F. A., \& Cedillo-Navarro, L. I. (2012). Toma de decisiones, estilos de comunicación en el conflicto y comunicación familiar en adolescentes bachilleres. Enseñanza e Investigación en Psicología, 17(2), 295-311. Recuperado de http://www.redalyc.org/revista.oa?id=292

Luna-Bernal, A. C. A., Mejía-Ceballos, J. C., \& Laca-Arocena, F. A. (2017). Conflictos entre pares en el aula y estilos de manejo de conflictos en estudiantes de bachillerato. Revista Evaluar, 17(1), 50-64. Recuperado de https://revistas.unc.edu.ar/index.php/revaluar

Markovits, H., \& St-Onge, M. J. (2009). Adolescents' and adults' internal models of conditional strategies for object conflict. The Journal of Genetic Psychology, 170(2), 135-150. doi: 10.3200/GNTP.170.2.135-150

Mejía-Ceballos, J. C., \& Laca-Arocena, F. A. (2006). Estilos de comunicación en el conflicto y confianza en las propias decisiones. Enseñanza e Investigación en Psicología, 11(2), 347-358. Recuperado de http:// www.redalyc.org/revista.oa?id $=292$

Munduate, L., Ganaza, J., \& Alcaide, M. (1993). Estilos de gestión del conflicto interpersonal en las organizaciones. Revista de Psicología Social, 8(1), 47-68. doi: 10.1080/02134748.1993.10821669

Pérez-Archundia, E., \& Gutiérrez-Méndez, D. (2016). El conflicto en las instituciones escolares. Ra Ximhai, 12(3), 163-180. Recuperado de http://www.raximhai. com.mx/Portal/index.php

Rahim, M. A. (1983). A measure of styles of handling interpersonal conflict. Academy of Management Journal, 26(2), 368-376. doi: 10.5465/255985

Rahim, M. A. (2001). Managing conflict in organizations (3a ed.). Westport, Connecticut: Quorum Books.

Rahim, M. A., \& Magner, N. R. (1995). Confirmatory factor analysis of the styles of handling interpersonal conflict: First-order factor model and its invariance across groups. Journal of Applied Psychology, 80(1), 122-132. doi: 10.1037/0021-9010.80.1.122

Ramírez-Landaeta, J. J., \& Borges-Grün, T. (2011). Adaptación y validación de un cuestionario de estilos de manejo de conflicto organizacional en una muestra de trabajadores brasileños. Revista Psicologia: Organizações e Trabalho, 11(1), 66-74. Recuperado de https://periodicos.ufsc.br/index.php/rpot

Rebollo-Catalán, A., Ruiz-Pinto, E., \& García-Pérez, R. (2017). Preferencias relacionales en la adolescencia según el género. Revista Electrónica de Investigación Educativa, 19(1), 58-72. doi: 10.24320/redie.2017.19.1.1022

Ricco, R. B., \& Sierra, A. (2017). Argument beliefs mediate relations between attachment style and conflict tactics. Journal of Counseling \& Development, 95(2), 156-167. doi: 10.1002/jcad. 12128

Rocha-Sánchez, T. E. (2008). La adolescencia: Período crítico en la construcción del sexo. En P. Andrade-Palos, J. L. Cañas-Martínez \& D. Betancourt-Ocampo (Comps.), Investigaciones psicosociales en adolescentes (pp. 15-44). Tuxtla Gutiérrez, México: Universidad de Ciencias y Artes de Chiapas y Universidad Nacional Autónoma de México.

Ross, R. G., \& DeWine, S. (1988). Assessing the Ross-DeWine Conflict Management Message Style (CMMS). Management Communication Quarterly, 1(3), 389-413. doi: 10.1177/0893318988001003007

Ruiz, M. A., Pardo, A., \& San Martin, R. (2010). Modelos de ecuaciones estructurales. Papeles del Psicólogo, 31(1), 34-45. Recuperado de http://www.papelesdelpsicologo.es

Saville, S. M., \& Swoap, R. A. (2006). Locus of control and conflict resolution: A comparison of early adolescents versus young adults. Asheville, North Carolina: Warren Wilson College. Recuperado de http:// 
www.warren-wilson.edu/ p psychology/saville.php

Sociedad Mexicana de Psicología (2010). Código Ético del Psicólogo (5a ed.). México: Editorial Trillas.

Thomas, K., \& Kilmann, R. (1974). Thomas-Kilmann Conflict Mode Instrument. Palo Alto, CA: Consulting Psychologists Press.

Weider-Hatfield, D. (1988). Assesing the Rahim Organizational Conflict Inventory-II (ROCI-II). Management Communication Quarterly, 1(3), 350-366. doi: 10.1177/0893318988001003005

Zwahr-Castro, J., \& Dicke-Bohmann, A. K. (2014). Who can be friends? Characteristics of those who remain friends after dissolution of a romantic relationship. Individual Differences Research, 12(4-A), 142-152. 